\title{
Older Adults' Attitudes to Self-Management of Health and Wellness through Smart Home Data
}

\author{
Julie Doyle, Niamh Caprani, Rodd Bond \\ NetwellCASALA, Dundalk Institute of Technology, \\ Dundalk, Ireland \\ julie.doyle@casala.ie,niamh.caprani@casala.ie, rodd.bond@netwellcentre.org
}

\begin{abstract}
Smart homes have significant potential to enhance the lives of older adults, extending the period of healthy ageing, through monitoring wellbeing, detecting decline and applying interventions to prevent or slow down this decline. In this paper we present results from interviews with 7 older adults who have been living in smart homes for over 4 years. Our aims were to 1) examine attitudes to living with sensors and AAL technology over time; 2) gather opinions on the usefulness of this data for supporting self-management of health and wellbeing and 3) evaluate the effectiveness of various visualization techniques for presenting sensor-based health and wellness data. Our findings show that older adults are interested in receiving feedback from sensor technology to support them self-managing their wellbeing. Potential beneficial information includes time spent inside and outside the home, walking time, sleep, activity, blood pressure and weight. This information needs to be enhanced by education and goal-setting and by representing data using visualisations that are simple and intuitive.
\end{abstract}

Keywords: Older adults; smart home data; wellness self-management.

\section{INTRODUCTION}

Globally, human populations are ageing [1] and there is a significant cost element to the health care of older adults. As a result there has been an elevated interest in understanding the factors that may support the maintenance of independent living and quality of life of older adults. Smart homes that support ambient assisted living (AAL) offer the capability to gather behavioural data from multiple sensing technologies around the home. One of the primary goals of smart homes is to support independent living, or ageing-in-place, allowing the older person to live in the place of their choice for as long as possible. Research has shown the potential for smart homes to detect acute health events as well as to detect changes in patterns of behaviour over time, that might indicate declining health [2-5]. Early detection, prevention and management ultimately enables more personally tailored interventions that can be delivered in a timely fashion, resulting in more individual, person-oriented care.

Analysis of smart home data can lead to very rich datasets of interest to many end-users, including older adults themselves, healthcare professionals, informal carers and family members. However, given the scale of data that smart homes produce, it is important to understand what data is useful, to whom, and what can be acted upon to improve health outcomes. Much research in this space has focused on the needs of healthcare professionals and family members, with comparatively less examining how older adults might use this information to self-manage their wellbeing [6], [7]. Research has also shown that the healthcare information needs of older adults, their family members and healthcare professionals differ [8], [9].

In this paper we introduce the Great Northern Haven smart homes, a development of 16 smart homes located in Dundalk, Ireland, where older people have been living since June 2010. We present data from interviews with 7 residents, the aim of which were to: 1) examine attitudes to living with sensors and technology over time - at the time of the interviews, residents had been living in the smart homes for 52 months; 2) gather opinions on the usefulness of this data for supporting selfmanagement of wellbeing and 3) evaluate the effectiveness of various visualization techniques for presenting sensor-based health and wellness data. We believe the value of this work lies in the longitudinal nature of the research, which allows us to go beyond looking at acceptance to issues surrounding older adults actually interacting with data generated from these sensors. This paper provides insights into how they would like to interact and how designers can support this.

\section{GREAT NorTHERN HAVEN}

The Great Northern Haven is a housing development consisting of 16 apartments, built purposely to support AAL for older adults. Each apartment is equipped with ambient sensor and interactive technology. The former includes PIR (passive infrared) sensors to detect movement, contact sensors on doors/windows and electricity sensing, supporting monitoring of patterns of behaviour over long time periods and detection of deviations from normal patterns of behaviour that might indicate the onset of illness. Interactive technology includes physiological sensing (blood pressure and weight) as well as iPads and smart TVs to feedback information on home security, energy and wellbeing to residents. It is important to note that technology is only one aspect of GNH. A number of social interventions also exist, including classes (from gardening and Tai Chi to iPad lessons), Men's Sheds, where men can work together on carpentry projects and a brokering service to make residents and other older adults in the surrounding area aware of services available to them.

Residents have lived in GNH since June 2010. Inclusion criteria included those over the age of 65 who had a health or housing need. We have gathered large amounts of data from 
sensors in the 16 apartments, and significant validation and analysis of data has taken place [5]. Algorithms have been developed to model patterns of daily behaviour and wellbeing [5], [11], [12]. A full description of the system architecture to gather and analyse data from $\mathrm{GNH}$, as well as some of the applications providing feedback to residents is presented in [5].

The residents of GNH have worked closely with the research team over the years in co-designing applications and interventions, with residents' needs at the forefront of the research. Initial focus groups with residents revealed that applications to help them manage home security and energy were important to them. Thus, residents have been receiving feedback on home security and home heating through iPad applications.

In terms of health and wellbeing, residents self-monitor their blood pressure and weight using Withings ${ }^{1}$ devices and their iPad. They also use a custom-made application, YourWellness, for self-reporting on their wellbeing, which provides feedback to them on their self-reported data over time as well as educational advice on healthy behaviours [13]. There is a 24-hour monitoring system in place, accessed through emergency buttons and cords placed throughout the apartment and alerts set up, based on ambient sensor data, to detect no movement and wandering.

Validating new algorithms is important to provide confidence in characterisation. Typically this is done by administering clinically validated questionnaires to participants being monitored at various time points [3] or asking participants themselves to label data, both of which can provide an approximate ground truth. The latter may be too obtrusive, and potentially erroneous. GNH residents complete a battery of health related clinically validated questionnaires every 6 months. These have been validated against various health metrics derived from the sensor data [6]. It was important to validate our algorithms prior to returning this information to older adults, to ensure the data was meaningful. To date, analysis of data has focused on a number of areas including activity within the home, time spent outside the home, night time activity, sleep patterns and disturbances.

The purpose of the interviews that are presented in this paper was to discuss with residents the type of health and wellness information we could present to them, what they were interested in and how they interpret such information presented in different visual formats. The results will inform the redesign of the YourWellness application, integrating sensor-based wellness data.

\section{RELATED WORK}

The idea of smart homes and monitoring technology is relatively new. One of the earliest introductions was by Dishman [14], who discussed the potential for such systems to collect data on behaviours, detect problems in a timely manner and support interventions. Given their potential to address many challenges associated with ageing, there is a vast amount of research within the smart home and AAL space, including to detect monitoring the onset of low mood or depression [15],
[16], motor and cognitive function [2], [17]; and patterns of activity [18]. Studies have ranged in design from short stays in artificial residences occupied by researchers, to deployments of smart home technology in the permanent homes of older adults.

Research at ORCATECH, Oregon Health and Science University, has comprehensively examined ambient sensing technology in the homes of older adults, primarily those with cognitive impairment. Their ISAAC project is large scale, with approximately 164 participants being monitored over a 5-year period [19]. Their research has focused on data analysis of nighttime activity, computer use and motor activity, including time spent outside the home [17], [18] and healthcare professionals' views on such monitoring technology [7]. From the older adult's point of view they have examined acceptability as well as willingness to share data and privacy concerns [20]. There is no reported research from ORCATECH on older adults interacting with their own data, or their views on this, though this may be a result of the cognitive profile of their participants.

In [2], the authors describe a study using AAL technology to monitor mobility in the home over a 6-month period, the aims of which were to determine correlations between sensor and self-reported data and assess the acceptability of sensor systems amongst older adults. However, the authors report a number of technology breakdown issues throughout the study, which prevented being able to generate patterns of activity and behaviour. This highlights the importance of robust validation work with sensor systems prior to delivering feedback on such to stakeholders. Chiriac et al. [4] describe their process for validating short-term and long-term rules of behaviour with data from 100 households, and conclude that both emergencies and long-term health problems, such as dementia, can be dealt with by their system. Robben and Krose [3] have also found significant associations between ambient sensor data, deployed in 9 older adults' homes, and a functional health assessment. Validation work at GNH has also shown significant correlations between features derived from ambient sensor data and anxiety, sleep quality, depression, loneliness, cognition, quality of life and independent living skills (IADL).

The vast majority of published research in this field has focused on issues surrounding data analysis to gauge useful information - a worthy topic given the vast amounts of data generated by such sensing systems. However, there is a need to move beyond demonstration projects and data analysis to interventions based on data generated from smart home technology. Some researchers have begun to examine how best to summarise and visualise relevant information for interested stakeholders, primarily clinicians and caregivers [8], [16]. On the other hand, comparatively little research exists on the older adult's viewpoint on such technologies and in particular their reactions to the data generated from sensing systems. Where older adults are consulted, the research tends to focus on acceptability of sensor and AAL technologies and issues surrounding privacy and data sharing. Fewer studies have examined older adults' attitudes towards the information and feedback that AAL systems can provide, including how they interpret and use such feedback.

\footnotetext{
${ }^{1}$ http://www.withings.com/eu/
} 
Reeder et al. [21] conducted interviews with 7 older adults who had lived with sensor technology in their homes for 6 months, to assess perceived usefulness of sensor data for managing health, factors that affect this as well as perceived usefulness of visual displays of sensor data. To stimulate discussion regarding visual displays, the authors used example data regarding a potential falls scenario, presented as 3 different visualisations, of which bar charts were the preferred method. Technical difficulties prevented the authors from displaying participants' own data. However, as participants were not living alone, it is unclear how the authors distinguished between residents in terms of the sensor data. Interestingly, older adults perceived visual displays of data as useful for caregivers of older people, including family members and health professionals, in particular those who might experience cognitive decline. In contrast, we're particularly interested in how such technology and feedback displays can be used to support preventative wellness management, and by the older person themselves.

TigerPlace [22] consists of 32 private apartments each kitted out with wired and wireless sensors that monitor proximity, motion, listen for audio alerts made by residents, measure vital signs, track residents' gait (using the Microsoft Kinect) and detect cooking patterns with temperature sensors. This research detects functional decline and alerts on-site caregivers when necessary. Alexander et al. [23] describe research conducted at TigerPlace, the aim of which was to test the usability of a computer-based interface that presents data from ambient sensors to older adults, their clinicians and carers, through various charts and graphs. The authors report that the 5 older adults who carried out the usability test had issues using a mouse and difficulty using text boxes. The older participants felt that the data would be useful for their caregivers, however the authors do not discuss whether the older adult participants would want to interact with such data themselves.

A study presented in [10] involves the concept of monitoring how well older adults perform activities of daily living (ADLs) and presenting this information back to older people, their caregivers and clinicians. The study probed older adults on whether they would find various health care information useful, including a monitor for their medication and information on activity performance including making a cup of coffee and telephone usage. The authors used simulated data to probe responses; the older adults interviewed $(n=4)$ were not living with sensor technology and were fully functioning. Participants stated they would be interested in this type of information as they got older and that they would want to know why there were declines in ADLs so that they could

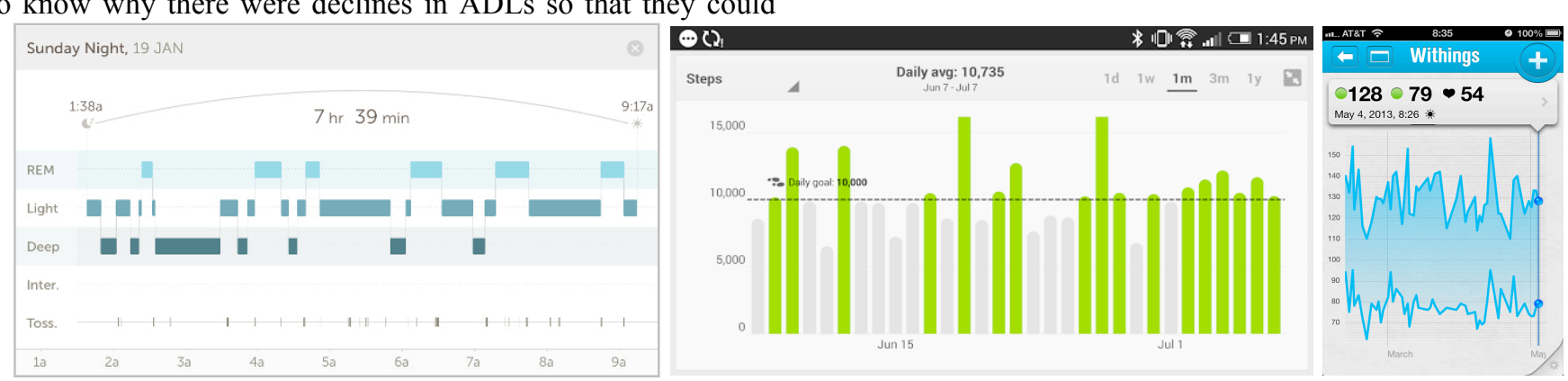

Fig. 1. Example visualisations shown to participants. Horizontal bar chart to display sleep activity (1a; www.mybasis.com), column chart highlighting achieved goals (1b; www.fitbit.com), and line graph displaying blood pressure readings (1c; www.withings.com).

'fix' any problems [10].

There are a number of questions that still need to be answered to understand how we might design feedback for older adults, to support them in managing their wellbeing based on sensor data. For example, are they interested in this data and if so what are they interested in? Under what circumstances will they find this information useful and actionable? What is the best way to present data to them? In the following sections we begin to explore these questions.

\section{STUdy Design}

This section outlines our study design, providing detail on participants and our methodology. Our aims were to 1) examine attitudes to living with sensors and AAL technology over time; 2) gather opinions on the usefulness of this data for supporting self-management of health and wellbeing and 3) evaluate the effectiveness of various visualization techniques for presenting sensor-based health and wellness data.

\section{A. Participants}

Seven participants from GNH were interviewed, including 5 men and 2 women. When we perform interviews or focus groups, we ask a subset of the residents to take part, so as not to overburden the residents too much with the research. With such a long-term project, it is important ethically to ensure participants are not over-burdened. Thus, while all residents have the sensor and interactive technology in their homes, they are not required to take part in all research activities.

Participant demographics are presented in Table I. Those interviewed ranged in age from 64 to 76 and had a wide range of health issues, including diabetes, heart conditions, chronic back pain and limited mobility. All take multiple medications. However, all participants are fully functioning and living independently.

TABLE I. PARTICIPANT DEMOGRAPHICS

\begin{tabular}{|l|l|l|l|l|}
\hline ID & Age & G & $\begin{array}{l}\text { Currently } \\
\text { monitoring }\end{array}$ & $\begin{array}{l}\text { Previous experience (before } \\
\text { GNH) with } \\
\text { computer/Internet }\end{array}$ \\
\hline 1 & 64 & F & Bp & Yes \\
\hline 5 & 74 & M & Bp, weight & No \\
\hline 11 & 64 & M & $\begin{array}{l}\text { Bp, weight, sleep } \\
\text { apnea }\end{array}$ & Yes \\
\hline 14 & 71 & M & Bp, weight & No \\
\hline 15 & 64 & F & Bp & No \\
\hline 20 & 76 & M & $\begin{array}{l}\text { Bp, weight, blood } \\
\text { sugar }\end{array}$ & No \\
\hline 25 & 75 & M & Bp, weight & No \\
\hline
\end{tabular}




\section{B. Methodology}

Each interview took place in the participant's home, lasted approximately an hour and was split into 2 sections. The interviews were audio recorded, transcribed, coded and analysed using thematic analysis.

\section{1) Section 1}

The first section asked questions around living with sensors and technology, including acceptability, obtrusiveness, how attitudes towards technology have changed over time, perceived benefits and drawbacks. For clarification, when we refer to the technology in participants' homes, we mean ambient sensors, physiological sensing and interactive devices, which are in all homes.

\section{2) Section 2}

The second part of the interview focused on health and wellness information derived from the sensors in the participants' homes, in addition to data from other sensors. For example, we introduced the idea of additional technology, namely an under-mattress bed sensor (that provides data as outlined in Table II) and a step-count or fitness device, such as Fitbit, as these are pieces of technology we have used in some studies with GNH residents and that we will continue to use in future studies. We began by asking participants if there was any particular information they would like to learn from the sensors regarding their health. We then showed some visualisations of health and wellness data derived from the sensors. Samples of these can be seen in Figures 1 and 2. Visualisations showing data over the space of short time periods (day/week) and longer time periods (number of months) were used.

We used these visualisations to probe what type of information would be of interest to them, how they might react to receiving such data and how useful it might be for managing their wellness now as well as in the future as their needs might change. The data focused on 3 areas of wellbeing, sleep, activity and physiological monitoring, which have been shown to be important in maintaining healthy ageing [11], [18], [24-26].

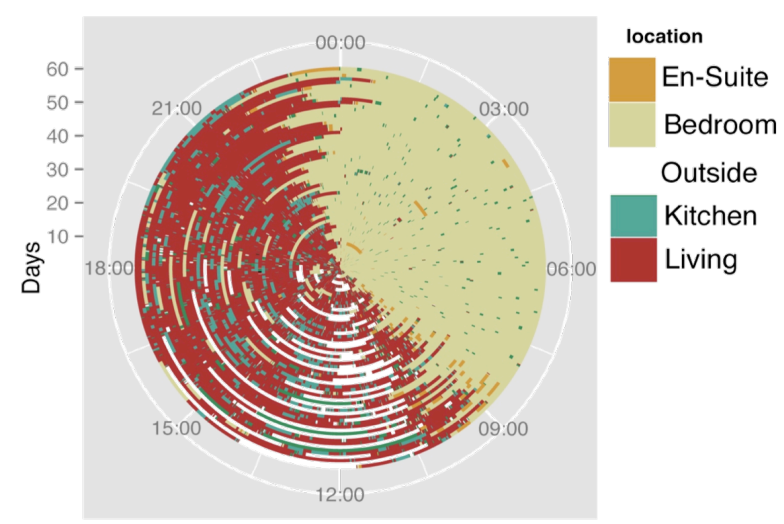

Fig 2. Clock plot displaying GNH resident home activity over a 60-day period.
TABLE II. SENSOR-DERIVED METRICS PRESENTED TO PARTICIPANTS

\begin{tabular}{|l|l|}
\hline Sleep & $\begin{array}{l}\text { Overall sleep quality score (as a percentage); number of bed } \\
\text { exits; number of tosses and turns; heart rate; respiration rate; } \\
\text { amount of time in bed; amount of time asleep and awake; } \\
\text { amount of time in light, REM and deep sleep. }\end{array}$ \\
\hline Activity & $\begin{array}{l}\text { Time spent inside and outside the home; room location; night } \\
\text { time activity; total time spent walking; number of steps. }\end{array}$ \\
\hline Physiological & Blood pressure; weight; heart rate \\
\hline
\end{tabular}

\section{RESUlTS}

In this section we discuss our results. Table III provides an overview of some of the specific interview questions asked to participants and outlines their responses. These are discussed in sections A-C below.

TABLE III. PARTICIPANT ATTITUDES

\begin{tabular}{|l|l|l|l|}
\hline & Yes & No & A little \\
\hline Are sensors obtrusive? are & 6 & 5 & 2 \\
\hline $\begin{array}{l}\text { Understanding that sensors } \\
\text { monitoring motion? }\end{array}$ & 6 & 1 & 1 \\
\hline Sleep data useful? & 1 & 6 & \\
\hline Room location data useful? & 4 & 1 & 2 \\
\hline Inside/outside data useful? & 4 & 1 & 2 \\
\hline $\begin{array}{l}\text { Physical activity data useful (step count } \\
\text { and walking time? }\end{array}$ & 6 & & 1 \\
\hline Emotional wellbeing data useful? changes in health & 4 & & 3 \\
\hline $\begin{array}{l}\text { Would alerts (of chatus) cause alarm? } \\
\text { status? }\end{array}$ & 7 & & \\
\hline Are regular patterns important? & 7 & & \\
\hline $\begin{array}{l}\text { Happy to share sensor/tech data with } \\
\text { others (including family/carers and } \\
\text { health professionals)? }\end{array}$ & 7 & & \\
\hline
\end{tabular}

\section{A. Living with Sensors and Technology}

During our interviews, we began by asking participants a number of questions relating to their opinions on living with the technology in their homes. As outlined in Table III, 5 of the 7 participants felt that the sensors were unobtrusive, stating that they rarely noticed them anymore. The red light from the PIR sensor was one reason provided for the sensors being somewhat obtrusive. P1 said, "In bed at night sometimes you roll over and one of them will ping. It's just a light will come on and that is really annoying. But apart from that I've just forgotten that they're there."

We also asked questions to determine whether participants fully understood what the sensors are monitoring. Given that they had been receiving sensor feedback through their iPads on home security, heating and energy, all were aware that energy was being monitored, as well as temperature and doors and windows being open or closed. In terms of the PIR sensors, 6 of the 7 participants understood that the sensors monitor movement. One participant thought they were also 'counting'. P11 said 'I'm not happy that they sort of count going in and out. The three of them (PIRs) are all at the doors. So they are counting the door opening and closing and in theory it can count the number of people coming in."

Further questions asked participants about their experience of living with the technology over the years, including any benefits or drawbacks they perceived. Safety and security were 
mentioned by each participant, with some stating the importance of these for emotional wellbeing. P20 said, "Before I came here I used to suffer about 4 panic attacks a year. I haven't had one panic attack since I moved here, my wellbeing here is $100 \%$."

Prior to moving to GNH, only 2 participants had previously used a computer and the Internet (Table I) and in our early interviews, many initially expressed an uneasiness regarding physically interacting with technology. However, all 7 participants reported actively using their iPads, including applications that had been custom-built for their homes as well as various other applications. The iPads appear to have been instrumental in participants' change of attitude towards technology. P5 said, "Ah since we got the iPad that was the start of the change in life and everything else." P25 said, "It's great. You're in touch with everything, anything you want." Weekly iPad classes are offered to GNH residents as part of the research programme, and residents reported using numerous applications including Skype, Spotify, FaceTime, Safari, email as well as various games.

Participants also commented on how their family members have been impressed by their new technology skills. P20 said "My grandson couldn't believe it, when he came in here first. Me. Doing this type of technology." P11 said "I'd see myself as a technophile. I like to see it (sensor feedback) working and get the benefit out of it." One participant, P1 reported using a smartphone, laptop, iPad and smart TV. However she said, " I've always hated it (technology) and I still hate it. I don't mind certain technologies obviously. I think it makes a great servant but I have no desire to live my life in a world dominated by it."

No participants reported concerns regarding privacy. During interviews in their first year at GNH, P1 and P11 had both expressed privacy concerns. Interestingly, these participants had previous experience with technology. The lack of privacy concerns may be due to the fact that residents are better informed about the usage of their data, including where it's stored and who has access to it. All said they would be willing to share the data with family members, a carer or clinician.

\section{B. Sensors and Health}

We began section 2 of the interview by asking participants if there was any particular information they would like to learn from the sensors regarding their health. Participants found it difficult to answer this question, until we began to use the visualisations as probes. The first topic of discussion centred around patterns. All participants felt having a regular pattern or routine was important, and reported typically going to bed, waking up at the same time each day, for example. Our data research over the years at GNH has demonstrated that this is the case with the majority of residents. The visualisations, for example the clock plot (Figure 2), were used to discuss how patterns might change, even subtly, over time and participants reported finding this information in general quite useful. P11 said, "I think seeing a pattern is very important. I'm talking about being able to observe and being able to know when you can seek professional advice, in different situations. I certainly think we need to know that. I need to know myself." Some participants said that if their patterns were changing, they would want to know why. P20 said, "I'd think it over and say I must be doing something wrong. And have a think about it." One participant, P5, commented on how it would be important to highlight improvement as well as decline. He said, "There are times when people come out of hospital and they say, well that's the end, its downhill from here. But the information (e.g. data showing improvement) can buck them up. It would lift you, of course it would."

In terms of usefulness of the sensor technology for monitoring health in general, there were mixed opinions. P1 said, "I will live alone for as long as I'm capable or until I die, whichever comes first. I really don't see how having extra input (from sensor data) is going to change that. I don't think it's going to prevent me tripping up, you know. But I wouldn't mind overlooking the data." Others referred to the alerts that had been set up, for example to detect no movement. P15 said, "I've never had to use it, but when you know it's there you know you can use it. It's security knowing that there is somebody at the other end.” P5 said “.. at least I'm moving, there's somebody who knows I'm mobile." P25 said, "Useful in the sense of if anything went wrong and you weren't doing the things that you normally do maybe they would alert you."

The interview then moved on to assessing the usefulness of the specific metrics outlined in Table II. Six participants said they would find sleep data useful. P5, who reports feeling exhausted even if he's been in bed for more than 8 hours, said, "It would be wonderful if it could tell me if I was asleep or awake." Participants reported strategies to try and sleep better, including stopping food and drink from $7 \mathrm{pm}$, or walking up and down stairs. P5 said, "I don't seem to be relaxed when I got to bed. I think I can't sleep before I even try." One participant, P11, suffers from sleep apnea - he felt that tosses and turns would be important information. P11 currently uses a machine, including a sleep mask, at night for sleep apnea. Data is recorded to an SD card, which he then sends off for analysis to the company who makes the machine, who then sends it to the doctor who requested it. However, P11 has never seen the data himself, even though he reports that he would find it very useful. Not all participants felt sleep information would be useful. P1 said "I don't need a sensor to tell me that I woke." However, P1 still thought some of the data could potentially be useful: "I could present that to the GP and say 'look I've got breaks in the pattern there, I think that needs to be looked at. If it was sleep apnea that would be a great guide for the GP, so yeah I can see that having some uses." P15 said "But how is it (data on sleep patterns) going to help? I mean, when you're asleep or supposed to be asleep, you still toss and turn, and you're still going to get out if you need to go to the toilet." This comment suggests that the participant wants actionable insight into his data, to show him what the data means and what steps to take to improve his patterns.

In terms of activity data, 4 participants thought information on time spent inside and outside the home would be useful, 2 felt it wouldn't, while 1 felt it would be somewhat useful. Only one participant felt that room location would be useful. P11 said "Say if you're going to the toilet a lot it would be very useful.. say if you had a problem." Other participants felt this information would be more useful to doctors. However, they 
felt that they are aware themselves of where they spend their time, so this information presented alone would not be useful to them personally. Four participants felt walking and step count data would be useful. Two felt it would be somewhat useful, though these participants both cycle and felt that this activity would be ignored by a pedometer. P11 said, "Step count is important as well when you're inside that you are getting up and doing things. You have here the daily goal, and to be able to count that and to see where you are is fantastic." The idea of settings goals was mentioned regularly. P1 said, "I like to keep challenging myself. Better myself with it." She also commented, "Its nice to have written confirmation of your achievements."

One participant, $\mathrm{P} 25$, reported finding none of the data useful at this point in time, though he felt education was important, particularly to help support what to do with the data. He said, "I can manage...but would I manage in two years time? That's a whole different ball game. Don't go away."

Participants talked about questioning why changes might occur. In relation to a potential drop in blood pressure values, P11 said, "That would be something that would be useful, to have a record of that. I wouldn't be aware (of what might cause the drop). But if you were following a pattern, perhaps you would become aware." It was generally felt that the data might prompt you to do something. For example, 4 participants said that if their time spent outside were to drop, that would encourage them to take a walk, or get outside. However, all participants commented that while seeing changes would be useful, they would also like information on how they could act on this data to improve their health, or what they should do in the case of a serious change. For example, we showed participants visualisations depicting a high blood pressure value and asked them how they would react to this. Four participants felt it would cause them alarm, while 3 didn't. P11 said, "You'd see how people would get worried about it. But I think its still important to know and in that situation that you get it looked at." P1 on the other hand said, "I would spend the whole day worrying."

\section{Understanding Visualisations of Health and Wellness}

The usefulness of smart home data to promote selfmanagement of health and wellness is reliant on how users interpret and understand visualisations of this data. We presented the participants with a series of graphs displaying sensor information to explore this. The participants were very comfortable reading column, line and pie charts to show daily, weekly and monthly activity (for example, Figure $1 \mathrm{~b}$ and $1 \mathrm{c}$ ). We were also interested in their understanding of more uncommon graphs, such as a sleep activity graph (Figure 1a) and clock plot (Figure 2), as well as others.

All participants interpreted the sleep activity graph correctly, "it makes an awful lot of sense" $\mathrm{P}(11)$, commenting on the length of time in REM, light and deep sleep over the course of the night and how they thought this would compare with their own sleep patterns. The participants showed less understanding of REM, compared to light and deep sleep, suggesting that information on these terms and how much of each is needed for healthy sleep is necessary. A sleep quality percentage score was also presented to the participants, which is calculated on the total time asleep, time in deep sleep, time in REM, time awake, and the number of awakenings. Again there was some ambiguity as to what this means; P11 asked, "Is $75 \%$ good quality sleep?" This suggests further information is needed to support user understanding e.g. through text, colour, icons.

Figure 2 presents the data as a clock plot. Clock plots are visualisations that we had used when showing this data to other potential stakeholders (such as clinicians) who found it a very useful way of showing patterns. The clock plot in Figure 2 uses colour to differentiate which room the resident was in at a particular time of the day. We wanted to know how older adults would interpret this information. We found that the participants understood the data in relation to activities over a day, for example P11 pointed out that the data showed the person went outside "rarely after 6 o'clock and never after 8 o'clock". The clock was better for depicting time than a linear block diagram and all participants said that they preferred the clock plot. However the clock plot showed 60 days of data, with each concentric circle representing a day, which was too many for the participants to easily read. Therefore we believe a weekly visualisation would be more appropriate.

The participants were presented with static visualisations, however one of the participants claimed that he would be interested in interacting with the graphs to explore more details about his data, "What I might like to do, if I have control of it, is manipulate it... look at how many times was my blood pressure over 140 say, how many times it was lower than 80. And if I'm informed I'd do a bit more work on it...Knowing how many times you're above the limit would be good" P11. Participants also said that they would find it useful to show their doctor their data if they believed that there was a problem, or to have as a record, "The visualisations are very good. It's useful to have it (the data), in a format...it would be useful to print out" $\mathrm{P} 11$.

It emerged that context was important for the participants to gain value from the smart home data, to see why patterns were changing as well as how. For example P20 said, "You'd wonder why. Why did I have a light sleep? Would it tell you why?" P11 suggested integrating the data coming from the sensors into one visualization would provide some context, "How, when and what's the connection. Say with that sleep graph, if you can find a common point between that and the bedroom (location data.)"

\section{Discussion}

\section{A. Living in Smart Homes - Effects on Technology Usage and Acceptance}

All participants mentioned some way in which they felt their lives had improved since moving into GNH. This was primarily due to the safety and security that the environment provided, in terms of 24-hour monitoring of alerts. Participants spoke about how this contributed to their emotional wellbeing. Having access to technology has also encouraged health monitoring, with participants regularly checking their blood pressure and weight, and generally making them more aware of 
managing their health. The benefits of technology go beyond health, however. Participants have used their iPads for connecting to family and friends, accessing information and for entertainment. Providing GNH residents with access to various education and classes has also provided benefit. Thus, ensuring a holistic approach, through the combination of technological, educational and social interventions, has led to these improvements. An interesting finding was that no participants reported privacy concerns, which is in contrast to other research [20], [27]. In some cases, such reports are from older people who have not lived with sensor technology (e.g. data gathered through focus groups) [27]. However, research at Orcatech found that $60 \%$ of older adults reported privacy concerns at the beginning of a study involving unobtrusive home monitoring, and that this increased one year after participation [20]. Our findings suggest that perhaps a level of trust has been built up due to a greater understanding of how the data is stored and managed, as well as giving the person control over how and with whom it is shared.

\section{B. Encouraging Self-Management}

There were mixed opinions on self-managing wellbeing and the usefulness of some sensor data, with P1 and P25 both stating that they don't see its utility for them at this point in time. This represents a challenge to encouraging selfmanagement of general wellness, amongst those who are not managing a chronic condition, for example.

One potential way to address this and to encourage selfmanagement from a preventative perspective this is to draw from the theory of reasoned action, which states that a person's intention to perform a behaviour is the immediate determinant and single best predictor of performing that behaviour, where intention has two basic determinants - attitude towards the behaviour and perceived expectation of important others [28]. Thus, a person will have strong intentions to self-manage their wellness if they evaluate it positively and if they believe it is important to others. Understanding whom the person views as 'important' (e.g. clinicians, family members) can help us to design a system that includes such people, allowing them to provide feedback to the person self-managing about the utility of such data and the benefits of self-management for prevention and maintaining wellbeing. To this end, we will integrate a communication feature within our application that will support a care network around the person. We think this will have two main benefits - to foster motivation and engagement and to help with understanding how to act on data (see next sub-section).

\section{Actionable Insights}

Participants repeatedly commented on how the data alone would not be sufficient for preventing health decline, with P1 highlighting that its not going to stop her from having a fall, and P15 wondering how information on bed exits will help reduce how many times you need to go to the bathroom for example. While this latter information may be useful to clinicians, who may want to understand nighttime behaviours and could prescribe a change in medication for example, this information alone may not be beneficial for the older person, beyond confirmation purposes. Participants also said changing patterns would prompt them to do something, though in some situations they were unsure what they would do. Some features of the data might be easier to act upon than others. For example, seeing a reduction in time spent outside might encourage you to get out. As P5 said, "You'd give yourself a bit of a shake and say come on, there's a bit of kick in you still." However, there are potential risks for older adults visualizing sensor data, including misinterpreting information or assuming it means something more significant. P 11 said, "A lot of the time you go up there (to the doctor), you might feel it's a trivial thing. If you see it on that (a visualization) you might think it's not too trivial otherwise it wouldn't show up. " Another potential risk is that the data may cause worry.

Increasing the utility of information provided by sensor systems might motivate the older person to keep an eye on it. Therefore it is critical that feedback to the older person should also include advice on how to act on data, as well as what to do in situations where a high alert is detected, such as with physiological data. We suggest three solutions that may support this, including providing education, setting goals for the person and connecting with the person's care network. Participants discussed the difficulty involved in getting access to information on how to manage health issues from their clinicians. P20 has suffered diabetes for a number of years, but was only recently told that he should always wear shoes in his home in case he cuts his feet and isn't aware due to the numbness he suffers as a result of the diabetes. Smart homes should provide social as well as technological interventions. We can also draw on the theory of self-efficacy [29] to increase an older person's confidence in their ability to play an active and important role in wellness self-management. This involves providing education not only on what healthy behaviours should be carried out, but the benefit of these.

\section{Importance of Context}

Visualisations of sensor data can identify patterns of behaviour and deviations from normal patterns. However, participants pointed out that the visualisations we showed did not explain why a pattern was changing. Other research has also discussed how the 'why' is missing from sensor data [10]. Some said they would visit their GP to try ascertain why changes were occurring. P11 said he would like the ability to manipulate the data - dig down deeper into certain elements, compare and contrast visualisations of different types of data to look for similarities. We observed some participants intuitively doing this while looking at the visualisations. P20 compared visualisations for overall sleep activity with bed exits: "You can see the dip there, like a helter skelter. And you see something similar there in the other graph. Very good." Suggestions for supporting context included allowing residents to annotate their data, as well as supporting comparisons of data from different sensors, as one may provide context for another. 


\section{CONCLUSION}

Smart home technologies can identify changes in patterns of behaviour that might indicate decline. Making relevant stakeholders aware of this information, in a timely manner, can potentially prevent or slow down the rate of decline. Given the vast amounts of data generated by smart homes, it is necessary to determine what information is useful and to address any challenges end users might have with interpreting and acting on the data.

In general our findings showed that people living over long time periods in smart homes find the technology not to be obtrusive in their day-to-day lives. Residents are for the most part interested in their own data and all are willing to share their data, once they retain control over what is shared and with whom. Participants reported a positive change to their wellbeing since interacting with technologies such as the iPad. However, they have not yet found benefit in ambient sensor data for managing health, as they are not currently receiving feedback. Potential beneficial information includes time spent inside and outside the home, walking time, sleep, activity, blood pressure and weight. However, it was clear that this information needs to be enhanced by education and goalsetting and by representing data using visualisations that are simple and intuitive. Our findings also demonstrate how smart homes, that combine technology and social interventions, can increase residents' feelings of security and wellbeing. One of the limitations of this study is the small number of participants who took part. However, there are very small numbers of older adults living with sensor technology over longitudinal periods.

Our future work will involve redesigning our existing wellness monitoring application, YourWellness [13], to include visualisations of sensor data. Our empirical research, combined with theories of health behaviour and behaviour change will support us in designing the application (determining what features to include and how to include them) and evaluating its effectiveness in supporting wellness self-management.

\section{REFERENCES}

[1] A.M. Hayutin, "Graying of the global population", in Public Policy and Ageing Report, 17, pp. 12-17, 2007.

[2] B. Reeder et al., "Testing a theory-based mobility monitoring protocol using in-home sensors: A feasibility study," in Res Gerontol Nurs, 6(4), pp. 253-263, 2013.

[3] S. Robben and B. Krose, "Longitudinal residential ambient monitoring correlating sensor data to functional health status" in $7^{\text {th }}$ International Conference on Pervasive Computing Technologies for Healthcare Pervasive Health), pp. 244-247, 2013.

[4] S. Chiriac, N. Roll, J. Parada and B. Rosales, "Towards combining validation concepts for short and long-term ambient health monitoring", in Pervasive Health, pp. 268-274, 2012.

[5] J. Doyle et al., "An integrated home-based self-management system to support the wellbeing of older adults", in Journal of Ambient Intelligence and Smart Environments, 6, 4, 2014.

[6] L. Walsh, A. Kealy, J. Loane, J. Doyle and R. Bond, "Inferring health metrics from ambient smart home data", in IEEE Bioinformatics and Biomedicine (BIBM)Workshop Proceedings, 2014.

[7] M.M. Davis et al., "A qualitative study of rural primary care clinician views on remote monitoring technologies", in Journal of Rural Health, vol. 30, pp. 69-78, 2014.
[8] M. Pavel, H. Jimison, T. Hayes and J. Kaye, "Technology in support of successful aging", in National Academy of Engineering - Bridge Issue on Technologies for an Aging Population, vol. 39, no. 1, 2009.

[9] B. Reeder, T. Le, H.J. Thompson and G. Demeris, "Comparing information needs of health care providers and older adults: findings from a wellness study" in MEDINFO, vol. 192, pp. 18-22, 2013.

[10] M.L. Lee and A.K. Dey, "Embedded assessment of aging in adults" in $4^{\text {th }}$ International Conference on Pervasive Computing Technologies for Healthcare (Pervasive Health), 2010.

[11] A. Kealy, K. McDaid, J. Loane, L. Walsh and J. Doyle, "Derivation of night time behaviour metrics using ambient sensors", in $7^{\text {th }}$ International Conference on Pervasive Computing Technologies for Healthcare (Pervasive Health), pp. 33-40, 2013.

[12] J. Loane, B. O'Mullane, B. Bortz and R.B. Knapp, "Looking for similarities in movement between and within homes using cluster analysis", in Health Informatics Journal, vol. 18, no. 3, 2012.

[13] J. Doyle, L. Walsh, A. Sassu and T. McDonagh, "Designing a wellness self-management tool for older adults - Results from a field trial of YourWellness", in $8^{\text {th }}$ International Conference on Pervasive Computing Technologies for Healthcare (Pervasive Health), pp. 134-141, 2014.

[14] E. Dishman, "Inventing wellness systems for aging in place", in Computer, vol. 37, pp. 34-41, 2004.

[15] S.M. Thielke et. al, "Associations between observed in-home behaviours and self-reported low mood in community dwelling older adults", in Journal of American Geriatric Society, vol. 62, no. 4, pp. 685-689, 2014.

[16] R.F. Dickerson, E.I. Gorlin and J.A. Stankovic, "Empath: A continuous remote emotional health monitoring system for depressive illness", in Wireless Health '11, San Diego, USA, 2011.

[17] J. Kaye et al., "Intelligent systems for assessing aging changes: Homebased unobtrusive and continuous assessment of aging", in Journal of Gerontology: Psychological Sciences, vol. 66, no. 1, 2011.

[18] J. Petersen, D. Austin, J.A. Kaye, M. Pavel and T.L. Hayes, "Unobtrusive in-home detection of time spent out-of-home with applications to loneliness and physical activity", in IEEE Journal of Biomedical and Health Informatics, vol. 18, no. 5, 2014.

[19] Intelligent Systems for Assessment of Aging Changes (ISSAC) project, http://www.ohsu.edu/xd/research/centersinstitutes/neurology/alzheimers/research/research-studies/issac.cfm, accessed January 2015.

[20] L. Boise, K. Wild, N. Mattek, M. Ruhl, H.H. Dodge and J. Kaye, "Willingness of older adults to share data and privacy concerns after exposure to unobtrusive in-home monitoring" in Gerontechnology, vol. 11, no. 3, pp. 428-435, 2013.

[21] B. Reeder, J. Chung, T. Le, H.J. Thompson and G. Demeris, "Assessing older adults' perceptions of sensor data and designing visual displays for ambient assisted living environments: An exploratory study", in Methods Inf Med, 53(3), pp. 152-159, 2014.

[22] M. Skubic, R. Guevara and M. Rantz, "Testing classifiers for embedded health assessments", in Impact Analysis of Solutions for Chronic Disease Prevention and Management, vol. 7251, pp. 198-205, 2012.

[23] G.L. Alexander, B.J. Wakefield, M. Rantz, M. Skubic, M.A. Aud, S. Erdelez and S.A. Ghenaimi, "Passive sensor technology interface to assess elder activity in independent living", in Nursing Research, vol. 60, no. 5, pp. 318-325, 2011.

[24] K. Manabe, et. al, "Sleep patterns and mortality among elderly patients in a geriatric hospital", in Gerontology, vol. 46, no. 6, pp. 318-322, 2000.

[25] L.E. Miles and W. C. Dement, "Sleep and aging", in Sleep, vol. 3, no. 3, pp. 119-220, 1980.

[26] S. Ancoli-Israel, "Sleep problems in older adults: Putting myths to bed", in Geriatrics, vol. 52, no. 1, pp. 20-29, 1997.

[27] L. Little and P. Briggs, "Pervasive healthcare: the elderly perspective", in Proceedings of the $2^{\text {nd }}$ International Conference on Pervasive Technologies Related to Assistive Environments, 2009.

[28] I. Ajzen and M. Fishbein, "Understanding attitudes and predicting social behaviour", Englewood Cliffs, NJ, Prentice-Hall, 1980.

[29] A. Bandura, "Self-efficacy: The exercise of control", NY, Freeman, 1997. 